\title{
Effect of Natural Polyphenols from Various Botanical Sources on Dentin Collagen Crosslinking and Stabilization Studied by Electron Microscopy
}

\author{
Hang Liu ${ }^{1}$, Jing Guo ${ }^{1}$, Donggao Zhao ${ }^{1}$, Yong Wang ${ }^{1 *}$ \\ 1. School of Dentistry, University of Missouri-Kansas City, Kansas City, MO64108 \\ * Corresponding author: wangyo@umkc.edu
}

Dental composite restorations face the lingering problem of short life time, particularly when bonded to dentin. One of the major culprits for dentin bonding to lose long-term stability is the degradation of demineralized dentin matrix over time. Generated in the bonding procedure by acid etching, this thin layer of denuded collagen fibrils is at elevated risk of hydrolytic and enzymatic breakdown due to myriad factors such as water sorption of dental resin and activity of matrix metalloproteinanses (MMPs). Therefore, collagen cross-linkers and MMP inhibitors have been considered as effective countermeasures to tackle the stability issue of dentin collagen and to eventually improve durability of dentin bonding. In this regard, our group has found that polyphenols, flavonoids-rich extract from grape seed, showed high efficacy as collagen cross-linker in clinically relevant settings [1,2]. However, this extract has high molecular weight and dark color (purple or red), thus inhibits infiltration and adhesive polymerization, respectively. There is a great need to evaluate other light color flavonoids-rich compounds from natural resources such as cranberry and green tea as collagen crosslinkers/MMP inhibitors in the similar clinically relevant manner. In this study, the effects of flavonoids/polyphenols from various botanical sources on the resistance of demineralized dentin collagen toward digestion of an exogenous bacterial collagenase and the activity of endogenous MMPs at the adhesive-dentin interface were evaluated using scanning electron microscopy (SEM) and transmission electron microscopy (TEM). The gold-standard cross-linker glutaraldehyde (GA) at comparable experimental conditions was used as control.

The occlusal crown was removed from human third molars to expose dentin surface. After acid etching with $35 \%$ phosphoric acid for 15s, the dentin surfaces were subject to the treatment with various natural compounds or GA at different concentrations for 30s to 1 minute. The treated dentin surfaces were digested by $0.1 \%$ bacterial collagenase solution (from Clostridium histolyticum) for 1 hour, after which the morphological changes of demineralized dentin collagen were investigated by a Philips XL30 ESEM-FEG (in both the secondary electron and back-scattered electron mode) and a Philips CM12 TEM. Further, selected treated dentin surfaces were bonded with SingleBond Plus adhesive. After the specimens were fractured to expose the adhesive/dentin interface, the MMP activity and morphological characteristics at the interface were gauged by zymography in combination with electron microscopy.

It comes as no surprise that the untreated demineralized collagen disappeared completely after $1 \mathrm{hr}$ of digestion. Surprisingly, the stabilizing effect of the control cross-linker GA at comparable experimental conditions was found to be almost non-existent within the same treatment times. In contrast, collagen treated with natural polyphenols from various botanical sources sustained the digestion at a different extent. Representative TEM results of polyphenols from tea theaflavins (TF) and grape seed extract (GSE) treated collagen layers are shown in Fig.1. A demineralized dentin layer was formed after acid etching (Fig.1A1). In a high magnification image (Fig. 1A2), the characteristic 67-nm banding pattern of collagen fibrils was obvious. This layer can easily be attacked by $0.1 \%$ collagenase and totally disappeared within 1 h of digestion (Fig. 1B). However, if treated by $2.0 \%$ TF for 30s, the digestion 
solution could hardly cause destruction to either integrity of the demineralized layer or characteristic pattern of collagen fibrils (Fig.1C). Demineralized layer treated with 2.0\% GSE showed the similar result after digestion (Fig. 1D). When the concertation was $0.4 \%$, both the TF (Fig. 1E) and GSE group (Fig. 1F) still showed existence of the demineralized layer. At a high magnification, however, the density of collagen fibrils at the bottom (Fig. 1F3) of the demineralized layer treated with $0.4 \%$ GSE became significantly lower than the top (Fig. 1F2) after digestion, which indicated the collagen might have been partially degraded. However, the demineralized layer treated with $0.4 \%$ TF was not affected by digestion (Fig. 1E2, 3).

\section{References}

[1] Y. Liu et al., J. Dent. Res. 92(8) (2013), p.746-752.

[2] Y. Liu et al., J. Dent. Res. 93(8) (2014) p.821-827.

[3] The authors acknowledge funding from NIH/NIDCR Grant R01 DE027049.
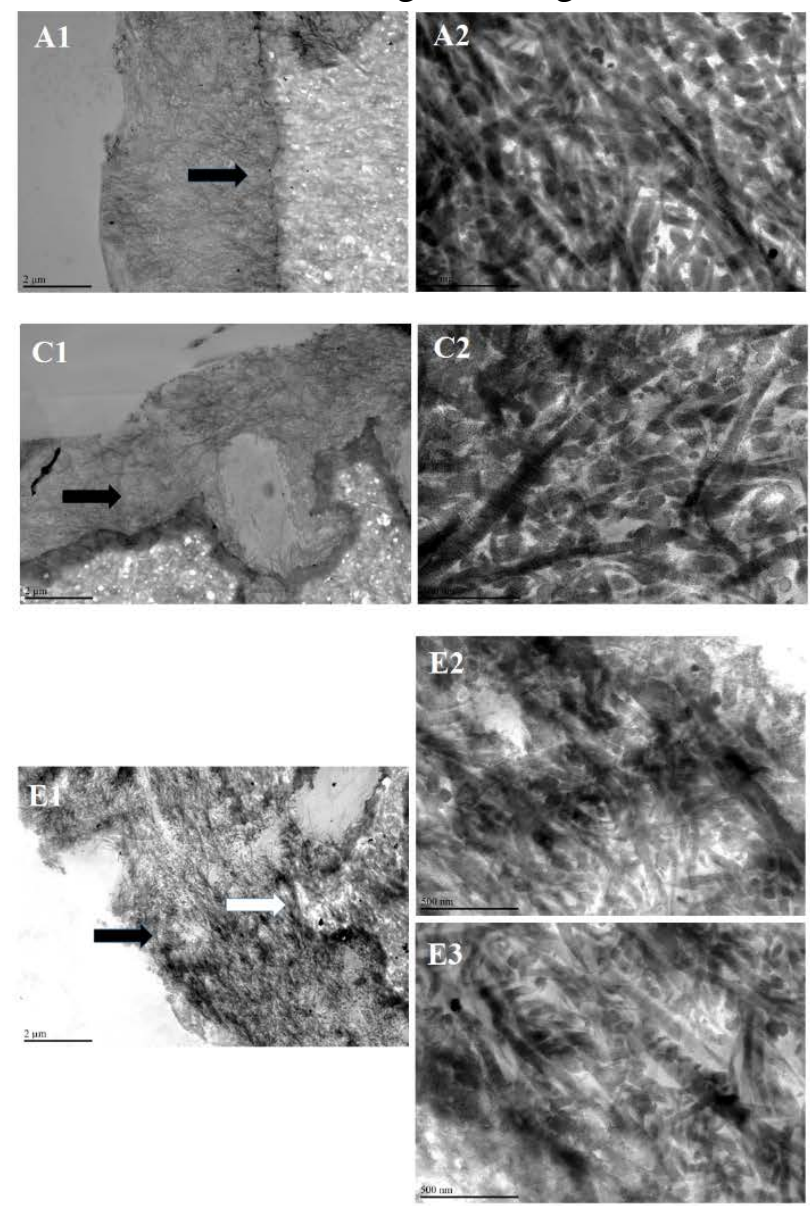
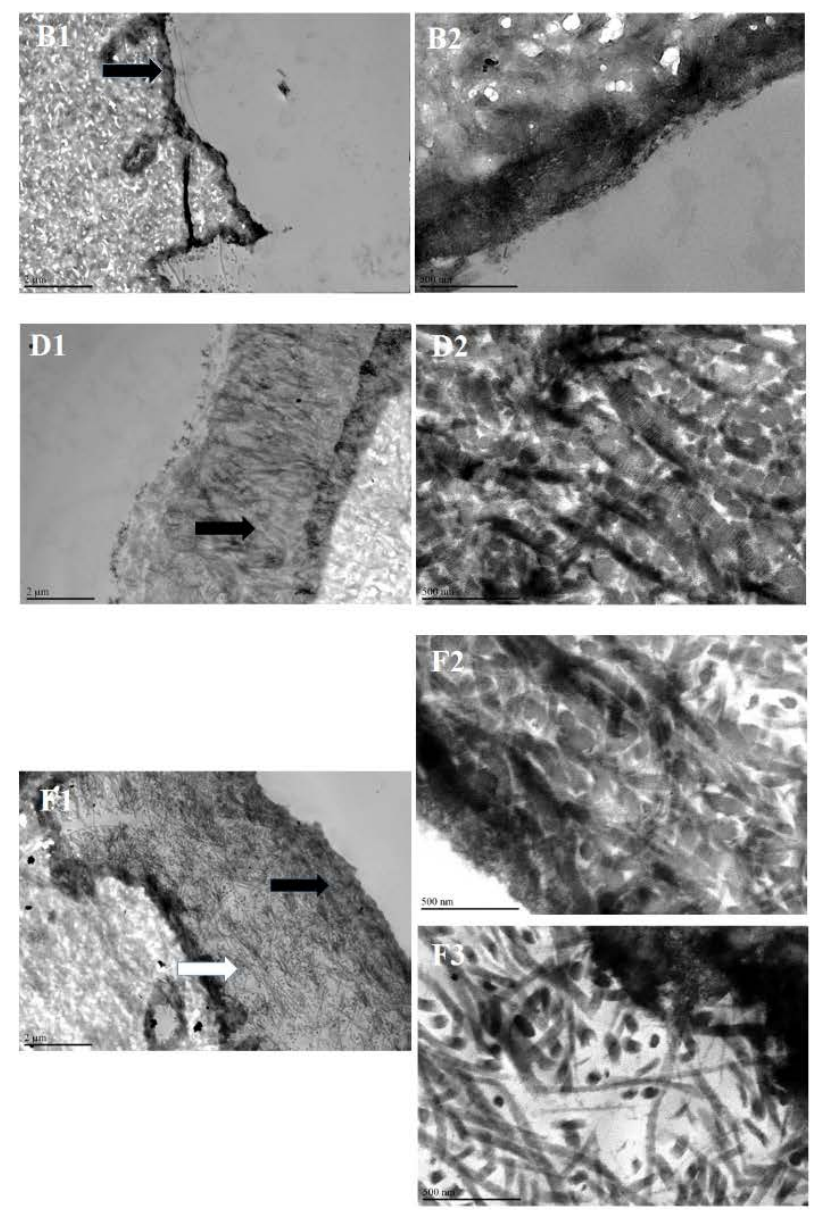

Figure 1. TEM images of demineralized dentin slabs: (A1) Untreated - without digestion; (A2) High magnification view of the arrow area in A1; (B1) Untreated - after digestion; (B2) High magnification view of B1; (C1) Treated with 2.0\% TF for 30s - after digestion; (C2) High magnification view of C1; (D1) Treated with 2.0\% GSE for 30s - after digestion; (D2) High magnification view of D1; (E1) Treated with $0.4 \%$ TF for 30s - after digestion; (E2) High magnification view of E1 at the black arrow; (E3) High magnification view of E1 at the white arrow; (F1) Treated with 0.4\% GSE for 30s - after digestion; (F2) High magnification view of F1 at the black arrow; (F3) High magnification view of F1 at the white arrow. 